\title{
You, Sledgehammer
}

\section{Lance Nizami $\odot$}

You concentrate
It does not break

You rise and pace

And still it does not break

You sit and stare and stare and stare again

It does not break; you break, and walk -

You let it go, and, strolling, find some other nut to crack

You find some niggling, smaller issue buried far inside your mind

But soon your mind's drawn back again, to wrestle the opponent -

You'll struggle fresh against yourself, to break the narrow box you think inside -

You'll shatter walls

That proof will break.

Independent Research Scholar

Palo Alto, California

USA

e-mail: nizamii2@att.net 